\title{
Testing ecological theories with sequence similarity networks: marine ciliates exhibit similar geographic dispersal patterns as multicellular organisms
}

\author{
Dominik Forster ${ }^{1}$, Lucie Bittner ${ }^{1,2,3}$, Slim Karkar ${ }^{3,4}$, Micah Dunthorn' ${ }^{1}$, Sarah Romac ${ }^{5,6}$, Stéphane Audic ${ }^{5,6}$,
} Philippe Lopez ${ }^{3,4}$, Thorsten Stoeck ${ }^{1 *+}$ and Eric Bapteste ${ }^{3,4 \dagger}$

\begin{abstract}
Background: High-throughput sequencing technologies are lifting major limitations to molecular-based ecological studies of eukaryotic microbial diversity, but analyses of the resulting millions of short sequences remain a major bottleneck for these approaches. Here, we introduce the analytical and statistical framework of sequence similarity networks, increasingly used in evolutionary studies and graph theory, into the field of ecology to analyze novel pyrosequenced V4 small subunit rDNA (SSU-rDNA) sequence data sets in the context of previous studies, including SSU-rDNA Sanger sequence data from cultured ciliates and from previous environmental diversity inventories.

Results: Our broadly applicable protocol quantified the progress in the description of genetic diversity of ciliates by environmental SSU-rDNA surveys, detected a fundamental historical bias in the tendency to recover already known groups in these surveys, and revealed substantial amounts of hidden microbial diversity. Moreover, network measures demonstrated that ciliates are not globally dispersed, but are structured by habitat and geographical location at intermediate geographical scale, as observed for bacteria, plants, and animals.

Conclusions: Currently available 'universal' primers used for local in-depth sequencing surveys provide little hope to exhaust the significantly higher ciliate (and most likely microbial) diversity than previously thought. Network analyses such as presented in this study offer a promising way to guide the design of novel primers and to further explore this vast and structured microbial diversity.
\end{abstract}

Keywords: Biogeography, Microbial diversity, High-throughput sequencing, Environmental rDNA sequencing, Protist

\section{Background}

Evaluating the patterns and processes of microbial diversity is central for understanding the ecology and evolution of life on Earth [1-3]. Currently, morphological and molecular methods have prompted a number of alternative perspectives on these issues. The extent of global dispersal versus levels of local endemism for microbial taxa [4-7], as well as the respective impacts of historical barriers to dispersal versus that of current conditions selecting among species in specific environments [8-11]

\footnotetext{
*Correspondence: stoeck@rhrk.uni-kl.de

${ }^{\dagger}$ Equal contributors

'Department of Ecology, University of Kaiserslautern, Erwin-Schrödinger-Straße 14, Kaiserslautern D-67633, Germany

Full list of author information is available at the end of the article
}

were debated to explain the distribution of these organisms. These perspectives have previously been difficult to evaluate for microbial eukaryotes because extensive diversity underlies many morphospecies and most species are recalcitrant to cultivation [12,13]. Traditional morphological and molecular methods have also imposed temporal and financial limits on collecting data on total diversity within and among communities [14,15].

The recent introduction of high-throughput sequencing (HTS) methodologies provided a way to push these limits to eukaryotic microbial diversity research [16-21]. It is now possible to quickly obtain hundreds of millions of sequences. However, the development of HTS was one of those events in which a nascent technology 
rapidly progressed far beyond our ability to best collect and analyze the data. This situation encouraged developments to justify which DNA region should be targeted for sequencing and which primer-pairs should be used, for example, in foraminifera [22], ciliates [17,23-25], dinoflagellates [26], fungi [27], bacteria [28] and archaea [29]. There has also been little effort put into effectively exploiting the resulting millions of short HTS sequences in a statistical framework, especially when placed into the context of previous studies. Computational steps are still a major bottleneck in molecular-based environmental studies. These shortcomings have hampered the identification of novel taxa, of distribution patterns, and their biological and ecological causes.

Sequence similarity network analyses, based on sequence similarity [30-35] offer an extension to sequence clustering analyses. They should not be conflated with co-occurrence networks [36,37]. Beyond a first step of clustering, sequence similarity networks allow biologists to perform fine-grained analyses of similarities between sequences, because they exploit the information provided by the topology of weighted connections between sequences within and between clusters. Such networks rely on methods from graph theory that have recently been adapted to address an increasing number of biological questions using large molecular datasets [30,31,38-43]. With such analyses, combining massive sequence data produced from numerous studies becomes feasible, and diversity patterns can be inferred that otherwise would not be apparent from individual studies alone. For example, a count of phylotypes in two separate datasets provides no information about which of these phylotypes are the same, while sequences from different studies can be directly compared in a single network analysis.

Phylogenetic analysis of short read data has made great strides in recent years, following the development of phylogenetic placement algorithms for incorporating short read data into a reference phylogeny (for example, $[44,45])$. Yet, multiple alignments and tree reconstructions with hundreds of thousands to millions of environmental sequences from HTS are either slow (when accurate) or inaccurate (when fast) [44]. Furthermore, a challenge in such tools still consists in developing appropriate visualization tools and metrics for analyzing distributions of reads on computed trees [44]. The network approach offers a powerful alternative in terms of comparative and visualization strategies (Figure 1), with the benefit of introducing several informative graph-based estimates describing the relationships between sequences (Table 1), thereby offering independent ways of analyzing the distribution of microbial organisms. In other words, sequence similarity networks allow the empirical testing of aspects of the theoretical framework of microbial ecology through the exploitation of network-based properties (Table 1).
Community clustering and Louvain community analyses [46] can be used to identify groups of similar sequences at various thresholds of sequence similarity. Assortativity analyses [47] can be used to define sets of sequences with distinctive characteristics. Path analyses [31] can be used to quantify divergence between sequences. All these measures offer an original path from graph theory to empirical analyses of the patterns and processes shaping microbial diversity.

In this study, we analyzed a network of 85,482 DNA and cDNA ciliate pyrosequences from the $\mathrm{V} 4$ region of the SSU-rDNA locus collected at eight European coastal sites from three different habitats (sediments, deep chlorophyll maximum (DCM), subsurface; for sampling details see $[18,48]$ ). We developed a protocol to: 1$)$ detect novel diversity in our new data in the context of existing sequence data from previous environmental diversity inventories (Additional file 1, Table S1) and cultured species; and 2) test ecological theories about ciliate dispersal at multiple evolutionary levels (from conspecific to more inclusive taxonomical units). We conclude that ciliates in European coastal areas-just like bacteria, plants and animals-are under strong environmental and geographical selection at intermediate geographical scale, and agree with previous observations rejecting global dispersal hypotheses [49-53]. We argue that such a diversity structure calls for specific improved sampling strategies in future microbial community surveys.

\section{Results and discussion}

\section{The large scope of network analyses}

Sequence similarity networks are inclusive graphs, easily accommodating substantial molecular datasets (typically millions of sequences, but billions are possible). They provide a unified comparative framework for these sequences, which can be analyzed with the methods and tools of graph theory [54]. In such graphs, the nodes represent the objects to be compared (here ciliate V4 sequences), each pair being linked by an edge if there is significant similarity between the two corresponding nodes (here a minimum \% identity (\%ID), E-value, length, and alignment cover spanning over the two sequences).

The resulting networks provide multiple lines of evidence to analyze genetic diversity in large molecular datasets at various sequence similarity levels, hence at various taxonomical levels, and consequently to test ecological theories (Table 1). First, thresholded sequence similarity networks effectively provide a first structure of the data by partitioning it, since the continuity and discontinuity of resemblances between sequences generally produces distinct subgraphs, called connected components (Figure 1A). When numerous sequences are highly similar, as is the case for V4 sequences, connected components grow to a very large size, forming Giant Connected 


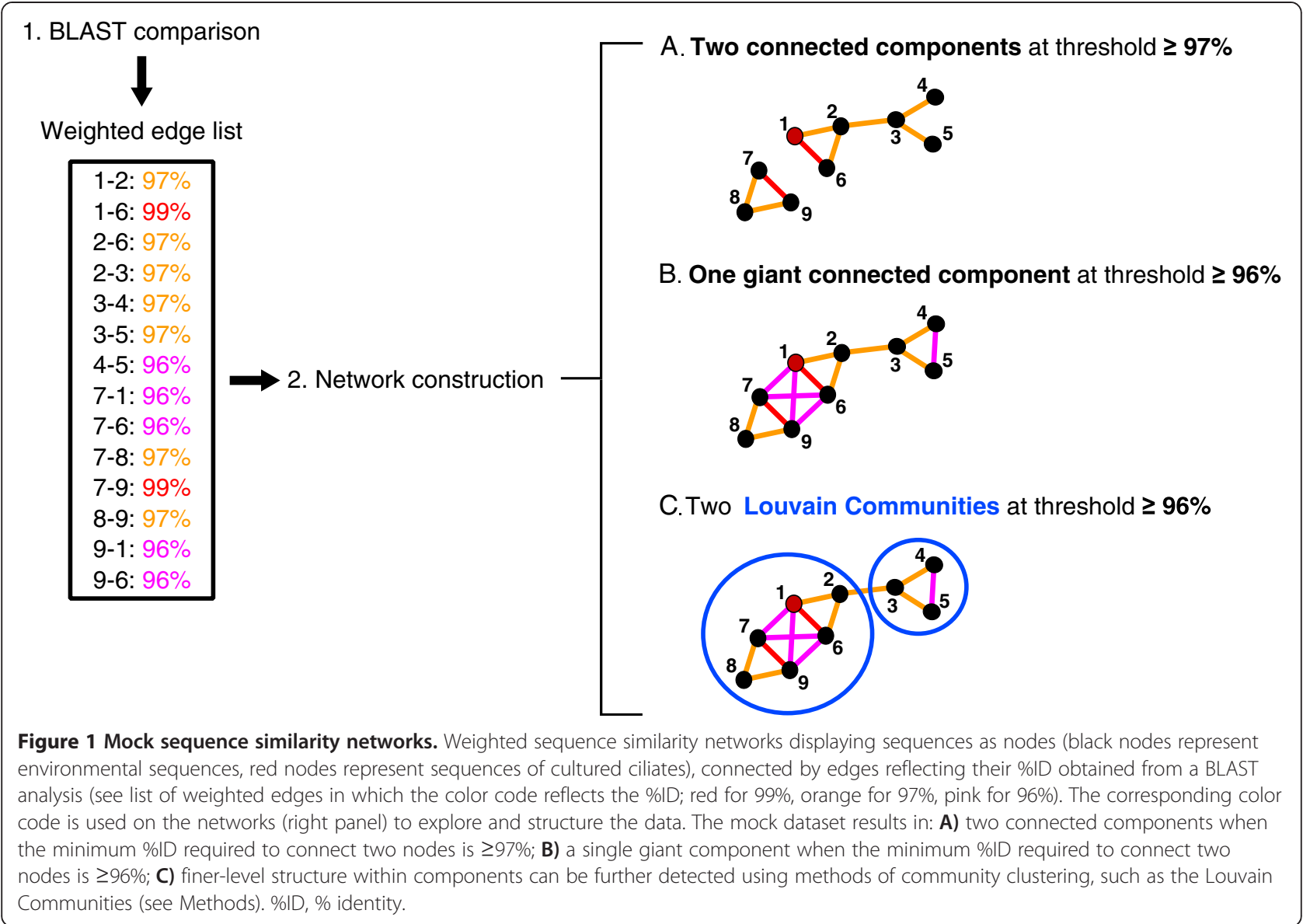

Table 1 Network methods and their adaptation to biological questions

\begin{tabular}{|c|c|c|}
\hline Network term & Term description & Biological meaning in this work \\
\hline Node & Single unit of a graph & A V4 sequence \\
\hline Edge & Connection between two nodes & Sequence similarity between two V4 sequences \\
\hline Assortativity & $\begin{array}{l}\text { Measure of the preferential connection } \\
\text { between a set of nodes of interest }\end{array}$ & $\begin{array}{l}\text { Evaluation of similarity of a set of sequences from the } \\
\text { same habitat or location (for example, if sequences } \\
\text { from one habitat are more similar to one another than } \\
\text { they are similar to sequences from other habitats, their } \\
\text { assortativity will be high. Environments with distinctive } \\
\text { similar V4 will have such a high assortativity). See Figure } 2 \mathrm{C} \text {. }\end{array}$ \\
\hline Closeness & $\begin{array}{l}\text { Measure of the centrality/peripherality of a } \\
\text { node in a network }\end{array}$ & $\begin{array}{l}\text { Measure of sequence divergence relative to the rest of } \\
\text { the dataset (for example, divergent sequences (with } \\
\text { respect to the rest of the dataset) have a low closeness } \\
\text { and tend to be more peripheral as they share less } \\
\text { similarity to other sequences). See Figure 2D. }\end{array}$ \\
\hline Shortest path & Shortest distance between a pair of nodes & $\begin{array}{l}\text { Measure to quantify the divergence between a pair of } \\
\text { sequences (for example, a long shortest path between } \\
\text { an environmental sequence and a sequence from a } \\
\text { cultured ciliate indicates a high divergence between } \\
\text { these sequences, since these sequences are not direct } \\
\text { neighbors in the graph). See Figure 2D. }\end{array}$ \\
\hline
\end{tabular}


Components [55] (GCC, Figure 1B). Second, connected components can be further partitioned to identify densely connected regions within them, known as communities in graph theory. In each community, nodes are more connected to other nodes within the given community than to external nodes (Figure 1C). We used Louvain communities [46] (LCs) of level 1 as the finer level of sequence similarity, that is, as the tightest clusters of similar sequences. We tested whether these two methods of graph partitioning (CC and LC implementation, respectively) returned groups of sequences from similar locations, depths, or lab cultures, or whether no such geographical, habitat or 'cultivability' structure was observed in the network. To do this, nodes in sequence similarity networks were labelled based on sequence properties. Preferential patterns of connections can then be analyzed in these labelled graphs, using assortativity estimates that quantify to what extent sequences with the same label (for example, from a given depth or location) connect with each other rather than with differently labelled sequences [47]. For instance, geographical structuring of the data produces (1) $\mathrm{CCs} / \mathrm{LCs}$ with sequences from only one sampling site (Figure 2A and C), and (2) CCs/LCs with sequences from multiple sampling sites, yet with detectable patterns of preferential connections between sequences from the same geographical location, if sequences from the same sampling site are more similar to one another than they are to sequences from other sites (Figure 2A and C).

A useful property of thresholded networks is that all sequences are not necessarily directly connected together within a CC or a LC. For instance, sequence 1 can be linked to sequence 2 , itself linked to sequence 3, while sequences 1 and 3 are not directly connected because they do not share a greater similarity than the stringency threshold used to construct the network. In that particular case, the connected component $(1,2,3)$ is a chain (Figure 1A). In practice, CCs (and to a lesser extent LCs) display a variety of mathematical and topological properties, ranging from chains to cliques (when all nodes are directly connected to one another), which can be exploited in comparative analyses.

Third, detailed analyses of the relationships between nodes provide additional network-based estimates of genetic diversity. In particular, the closeness of a node, quantifying its location in the graph, and the number of edges separating two given nodes can be computed [56] to compare the centrality of environmental sequences with that of sequences from cultured ciliates, and to measure their relative dissimilarity, when environmental sequences are located more than one edge apart from any sequence from cultured ciliates (that is, if sequence 1 in the chain described above was a sequence from an organism in culture and sequence 3 was a sequence from an environmental sample; these two sequences could be considered as divergent with a distance $=2$ for that threshold).

In short, sequence similarity networks offer a global and inclusive framework, that allows displaying and comparing novel data with pre-existing samples and, therefore, to progress towards integrated comparative analyses of multiple data sets. Using these tools, we analyzed (1) how environmental SSU-rDNA projects have expanded our former knowledge of genetic diversity that was based on cultured ciliates, (2) how the results of different environmental SSU-rDNA surveys of microbial eukaryotic diversity compare in terms of genetic diversity, while (3) enhancing our understanding of the actual global diversity of ciliates and of its ecological structure.

\section{Extensive novel diversity of environmental ciliates}

Previous environmental diversity studies $[19,21,57,58]$ have shown that it is specifically the large proportion of low-abundance taxa in a microbial consortium, in which we find most of the novel diversity. Figure 3 shows the GCC of the DNA and of the cDNA networks constructed at the most inclusive threshold of $\geq 85 \%$ similarity. Densely connected regions (LCs of the GCC) of similar sequences are replaced by supernodes for display purposes. These supernodes are colored based on the percentage of sequences they comprise either from cultured organisms ( $\mathrm{t}-2)$, from cultured and environmental sequences obtained before the BioMarKs project [48] by Sanger sequencing $(t-1)$, or that were obtained by the BioMarKs project [48] ( $\mathrm{t}$ ) using 454 pyrosequencing. In other words, Figure 3 reveals the similarity relationships between sequences from cultured organisms and from an increasing number of environmental projects.

Ciliate sequences obtained by BioMarKs (represented by all non-pink LCs in the right graph, Figure $3(\mathrm{t})$ ) substantially increase the diversity that was known from sequences of described organisms (represented by all non-green LCs in the left graph, Figure $3(\mathrm{t}-2)$ ). For cDNA sequences, the number of LCs increases 1.4 fold and for DNA 2.3 fold. When all ciliate sequences from the environmental reference database are also considered in the GCC network analyses, we find that a large proportion of BioMarKs diversity was, in fact, discovered previously in environmental molecular diversity surveys (non-pink nodes in Figure $3(\mathrm{t})$ and non-green nodes in Figure $3(t-1)$, respectively). The colors of these GCCs show that environmental projects conducted before BioMarKs mostly detected ciliates sequences that were either from the same LCs as sequences from cultured organisms, or from previously untapped LCs neighboring these communities. This observation means that rather central LCs with sequences from cultured ciliates were further enriched with environmental representatives (yellowish communities in the left graph turning 


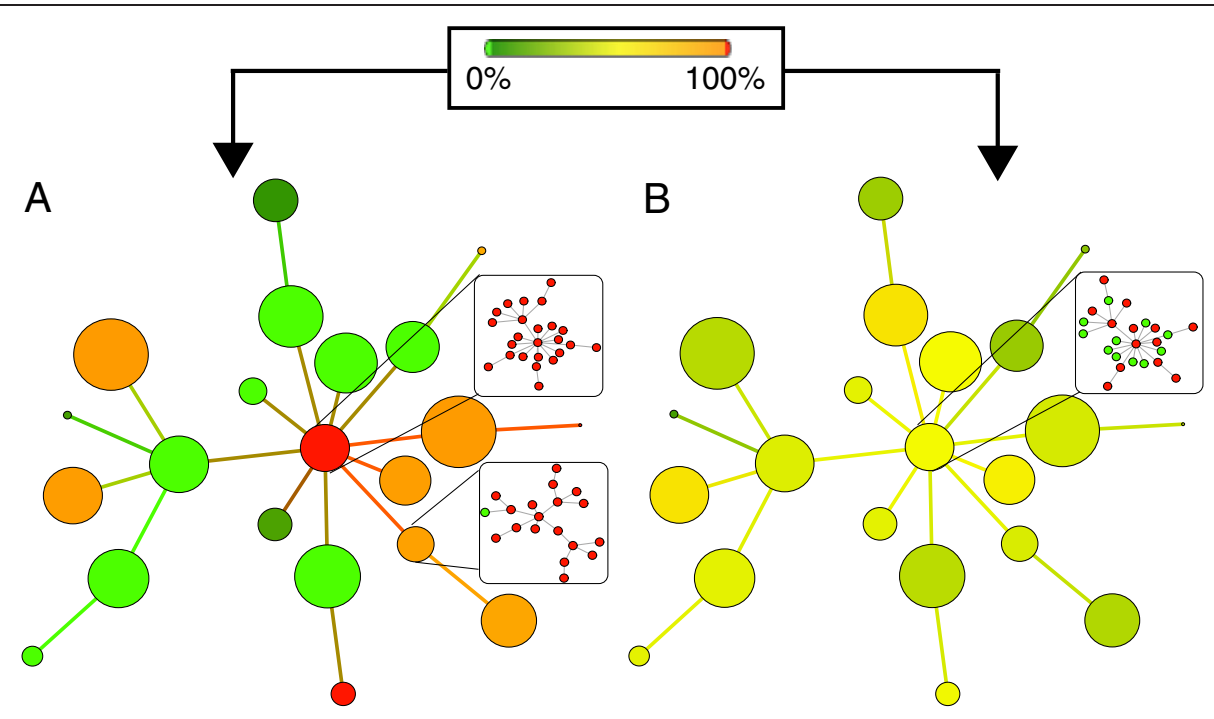

C
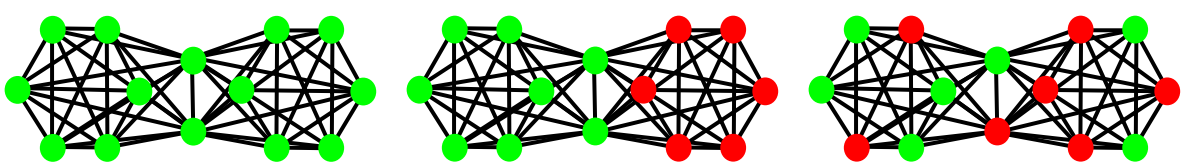

D

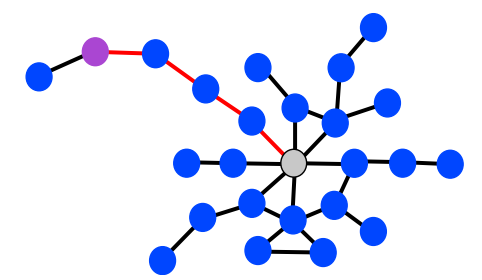

Node exhibiting high closeness

- Node exhibiting low closeness

— Shortest path between highlighted nodes

Figure 2 Mock sequence similarity networks displaying endemism and cosmopolitan distribution of ciliates. A) Schematic pattern of a GCC colored to reflect the origin of sequences in case of endemism. Each supernode corresponds to a LC consisting of a group of sequences. Exemplary LC composition is described in the insets in squared boxes. Each node color in these insets corresponds to a given hypothetical sampling depth (that is, red for depth A, green for depth B). Each supernode color represents the proportion of sequences from different sampling depths in a LC (see color bar ranging from green (100\% sequences of depth B) to red (100\% sequences of depth A). B) Schematic pattern of the same GCC colored to reflect the origin of the sequences in case of a cosmopolitan distribution of ciliates. Same color code as above. Intermediate colors of most LCs reflect the fact that highly similar sequences were detected at different depths. C) Schematic pattern of $\mathrm{CCs}$, their node colors reflecting the origin of the sequences. As above, each color corresponds to a given sampling depth. Some structure is visible when either all similar sequences originate from the same depth (left), or when sequences from a particular depth cluster together within the component (middle). In case of a widespread dispersal across sampling depths, no structure with regard to the color code can be observed (right). D) Schematic pattern of a CC, describing closeness and shortest path. Two distinct nodes are highlighted. One in the center (grey) and one in the periphery (lilac) of the CC, exhibiting high and low closeness, respectively. Red edges connecting the highlighted nodes display the shortest path between these two particular nodes. See Table 1 for further explanations. CCs, connected communities; GCC, giant connected components; LC, Louvain communities.

more orange in the middle graph), and at the same time novel communities were discovered during these early environmental projects (green communities that were lacking any cultured member in the left graph turning yellowish in the middle graph).

The inclusion of environmental sequences from BioMarKs continued this expansion in the description of genetic diversity in ciliates, identifying more environmental representatives of previously known communities (yellowish and orange communities of the middle graph turning light blue in the right graph), and detecting novel communities (green in the middle graph, dark blue in the right graph) especially at the periphery of the graph. Figure 4 gives a more precise overview of the novel ciliate diversity revealed by the sequence similarity networks at the $\geq 85 \%$ threshold. Our BioMarKs data added eight distinct and previously unknown LCs to the cDNA dataset and twelve to the DNA dataset (Figure 4). 


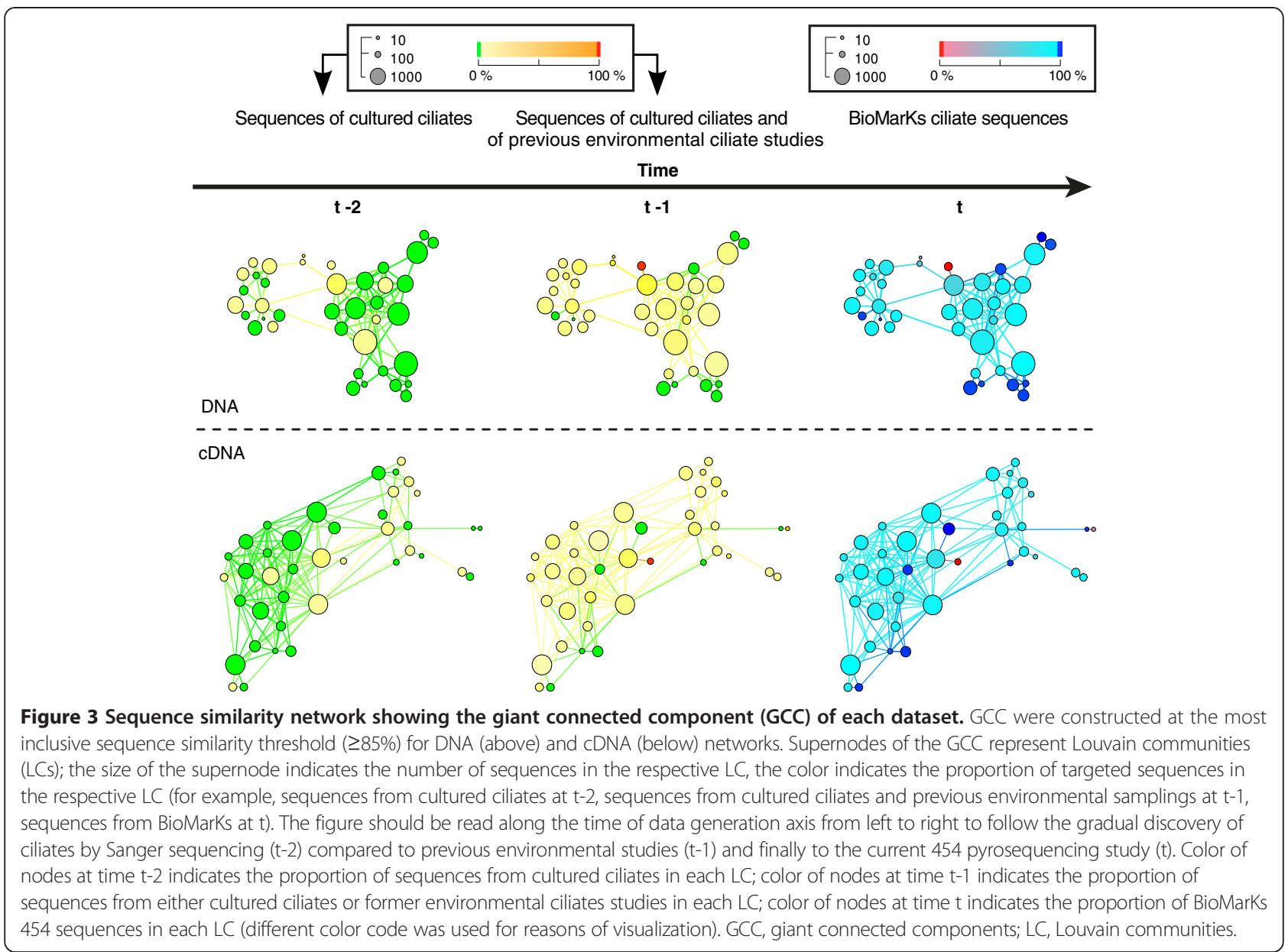

The composition of these LCs does not imply the presence of a specific hotspot for the detection of novel ciliate diversity with regard to location or habitat (Figure 4). Although most of the LCs could be assigned to the class Spirotrichea, the low sequence similarities to the closest cultured references indicate that the taxonomic assignment should be taken with care.

Moreover, the structure of the sequence similarity networks also enabled us to quantify and characterize the novel diversity detected by $\mathrm{CC}$ analyses. At all similarity thresholds, we detected components that exclusively consisted of BioMarKs sequences (Additional file 2: Figure S1). For example, at $97 \%$ sequence similarity, a suggested approximation to discriminate ciliate species based on the V4 fragment [24], 60\% of the cDNA components and $45 \%$ of the DNA components did not include any reference sequence, thus representing previously unknown ciliate diversity.

The divergence between environmental sequences and sequences from cultured ciliates was further analyzed by network path analyses (Table 1) of CC and LC. These analyses also provided evidence that environmental sequences correspond to (1) already known sequences or are highly similar to such sequences $(16.6 \%$ DNA and $10.5 \%$ cDNA sequences at distance $=1$, thus directly connected to a cultured ciliate sequence in the CCs at $\geq 97 \%$ similarity, see Additional file 3: Figure S2) but also to (2) novel sequences, expanding the known genetic diversity of ciliates (83.4\% DNA and $89.5 \%$ cDNA sequences at distances $>1$, thus indirectly connected to cultured ciliates sequences in the CCs at $\geq 97 \%$ similarity, see Additional file 3: Figure S2). These novel sequences only share direct connections to other environmental sequences, and are observed even at very inclusive stringency thresholds $(60 \%$ DNA sequences and 58\% cDNA sequences at $\geq 85 \%$ similarity, see Additional file 3: Figure S2). Hence, many environmental sequences cannot be directly assigned to formerly described lineages. Results are similar for LCs (Additional file 4: Figure S3).

Likewise, the analysis of closeness values, contrasting the centrality of environmental sequences with that of sequences from cultured ciliates, provides similar conclusions. The centrality of a sequence reflects its similarity to all other sequences in the graph. The more central one sequence is, the higher its closeness and the least divergent it is with respect to all other sequences in the 


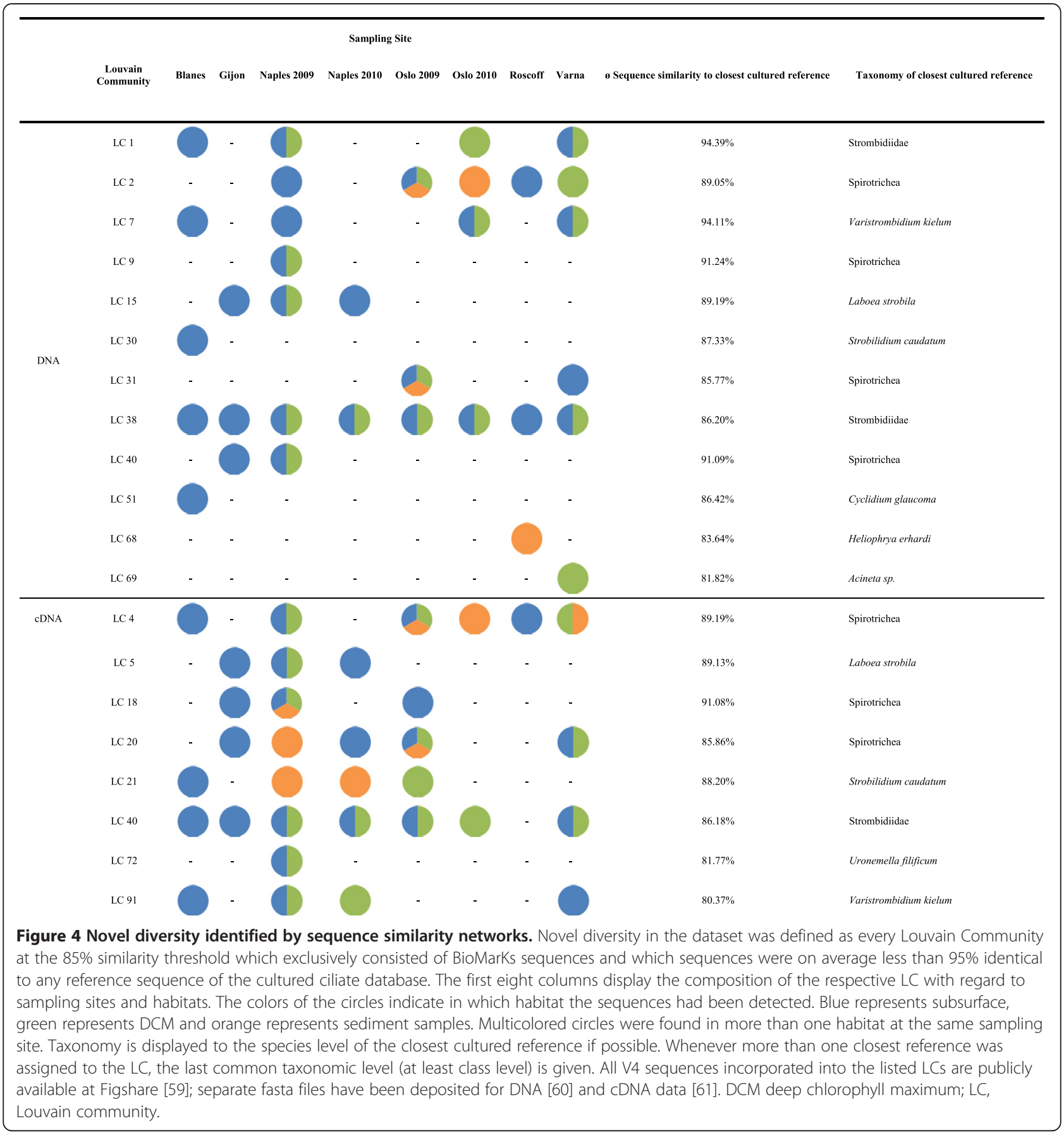

network. Environmental sequences, which are more divergent to sequences from organisms known from cultures, occupy the periphery of the graph. In all networks and subgraphs, sequences from cultured ciliates are more centrally located than sequences from environmental projects (Additional file 5: Table S2 and Additional file 6: Table S3). Environmental SSU-rDNA studies are thus expanding the description of genetic diversity beyond that of known sequences at all taxonomical levels, if one assumes that decreasing similarity between sequences corresponds to increasing evolutionary distance between ciliates. The only exception concerns CCs at the $\geq 90 \%$ similarity threshold, in which no significant differences between the closeness of sequences from cultured ciliates and from environmental studies before BioMarKs were observed. Yet, when BioMarKs sequences are considered in this particular analysis, the location of cultured ciliate sequences in central positions of CCs at $\geq 90 \%$ similarity is statistically supported $(P<0.01$, Kolmogorov-Smirnov test with unilateral option, see Methods; Table S2). In 
summary, all networks indices (paths, closeness, CCs and LCs) point to the same conclusion: network analysis unravels novel ciliate diversity, discovered by the current survey strategy.

Furthermore, our results provide an 'historical' perspective on the progress in diversity studies of ciliates. On one hand, they show an inherent conservative bias of environmental SSU-rDNA surveys that rediscovered 'more of what was already known' in terms of genetic diversity. On the other hand, they show that even after two centuries of microscopy studies and about 20 years of molecular diversity analyses, HTS sequencing approaches still reveal substantial amounts of novel diversity. Sequence similarity networks provide us with the tools to recognize the degree of this novel diversity.

Yet, how we increase our knowledge of genetic diversity of environmental ciliates clearly depends on our background knowledge: environmental diversity surveys using primers derived from cultured organisms catch in first place more of the well-known described diversity [62], rather than detecting novel peripheral groups in the networks. This suggests that the use of different sets of primers, for example, designed from alignments of the environmental sequences in these peripheral communities (Figure 4), may be an efficient opportunity to expand further the discovery of novel, divergent groups of ciliates. More precisely, groups of sequences, such as cliques [63] or peripheral sequences can be easily extracted from sequence similarity networks, producing sets of sequences with shared similarities. These sequences can be further aligned with each other to identify specific shared divergent regions, which can become new primers for upcoming analyses. This strategy could complement the extraction of SSU-rDNA genes from PCR-free shotgun metagenomic datasets, and, within these less taxonomically biased datasets, ease the selection of SSU-rDNA genes with sequence similarities to (groups of) divergent V4.

\section{Geographic structuring of ciliate communities}

We mapped the geographic distribution of our sequences in similarity networks to test the ecological hypothesis that ciliates are cosmopolitan organisms. Assortativity analyses were used to quantify to what extent sequences from any location and depth have close homologs in more than one environmental sample of the data set. Since similar sequences are directly connected in our graphs, the larger the environmental distribution of ciliates with similar sequences, the lower the assortativity of sequences from a given location or from a given depth in the graph (since ciliates from different locations/depths with similar sequences directly connected in the graph display different labels, Figure 2B). By contrast, if ciliates with similar sequences preferentially occupy one given depth or one given location, their sequences will tend to form clusters which exclusively group sequences from that given depth or location. Sequences of ciliates with restricted geographical or habitat distribution will tend to connect with each other in our networks, and the assortativity of their respective labels will be high (Figure 2A). Hence, the proportion of connected components with significantly higher assortativity values than expected by chance for any location or depth in our graphs indicates to which extent we observed some geographical or habitat structure in the distribution of ciliates in our data set. The majority of the three tested labels for habitats, and of the eight tested labels for locations, were significantly more assortative in the analyzed networks, indicating a notable geographical and habitat structure of BioMarKs ciliate sequences (Additional file 7: Table S4). The structuring effect is especially emphasized in the cDNA dataset for networks generated at thresholds $\geq 95 \%$ sequence similarity (Additional file 7: Table S4).

Furthermore, the global dispersal hypothesis of individual taxa was not supported by our data. On the contrary, only a maximum of $2 \%$ of all CCs at the $\geq 99 \%$ similarity threshold include sequences from all sampling sites (Figure 5). Figure 5 shows the abundance of sequences for each CC (results for LC were similar, see Additional file 8: Figure S4), the number of locations in which sequences from these clusters were found (occurrence), and whether these sequences were evenly distributed across the locations in which they were detected, or rather dominant at some specific location (evenness). The vast majority of all components (between $92 \%$ and 95\%) is restricted to four or fewer sampling sites and is found at low abundance. Only a few groups of ciliates were found in all samples at high abundance with an even distribution of sequences. This further fuels the moderate endemicity hypothesis for protists [7], which was also supported in previous morphospecies-based studies $[52,53,64,65]$ as well as in environmental genebased diversity inventories [49-51]. Oxygen and salinity were identified in these and other studies as major dispersal barriers for protists (for example, [66]) and also for bacteria [67]. Indeed, oxygen-depletion may be a major factor that distinguishes the water samples from the sediment samples in our BioMarKs data set (most sediments generally become anoxic within a few millimeters below the surface [68]). Likewise, salinity differences were noteworthy among the BioMarKs sampling sites, ranging from 16.73 psu (practical salinity units) in the Black Sea to 37.93 psu in the Mediterranean Sea [48]. Additional possibilities are discussed elsewhere as potential dispersal barriers for microbes; for example, constraints in active and passive dispersal $[11,69]$, and also the success rate of colonization (establishment of a stable population) in the new environment [70]. The latter 

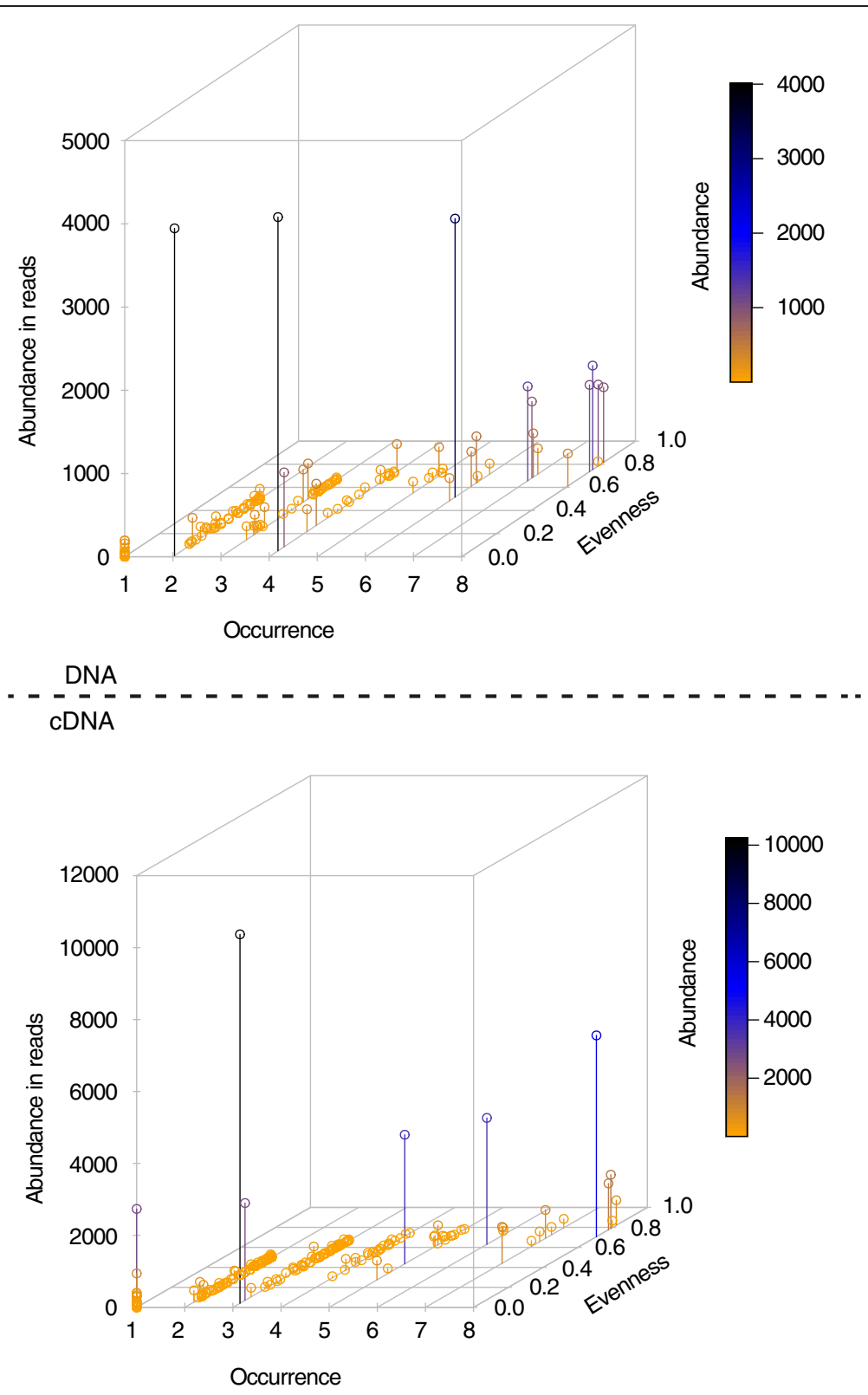

Figure 5 Three-dimensional plots displaying abundance, occurrence and evenness of sequences in connected components. Plots are based on DNA (above) and cDNA (below) networks at the most exclusive sequence similarity threshold (299\%). Each dot (339 for DNA; 593 for CDNA) represents one $C C$, hence one conspecific group of ciliate sequences. Color and position of a dot on the $y$-axis indicates its abundance (the number of sequences in the CC). Occurrence refers to the number of sampling sites (maximum = 8) at which sequences of the CC were detected. Evenness (estimated as Simpson index) describes whether sequences of a CC are homogenously distributed across different sampling sites ( $\mathrm{SI}=0$ indicates an uneven distribution, SI close to 1 indicates an even distribution). LC analyses revealed similar results (see Additional file 8: Figure S4). CC, connected components; LC, Louvain communities.

is influenced by a wealth of biotic and abiotic processes [71]. Although there may be limits to the biogeography of many protists, ciliates, like bacteria [72], may have a common core of diversity present at very low levels, a phenomenon that could be tested with HTS for example. Finally, while geographically widely dispersed components include slightly more abundant sequences, not all abundant sequences are widely dispersed. For instance, the component with the highest abundance in Figure $5(n=10,255)$ includes sequences from only three different sampling sites.

\section{Habitat selection on ciliate populations}

Besides network components that are geographically restricted to one or a few specific sampling sites (Figure 5), the effect of environmental selection is also shaping 
ciliate community composition (Additional file 7: Table S4, discussed above). The GCC of the DNA and cDNA networks at $\geq 85 \%$ similarity clearly illustrate this situation, in spite of their low stringency threshold (Figure 6), ciliates in sediments exhibit sequences that are different from ciliates detected in the water column (subsurface and DCM). While subsurface and DCM sequences are often found in the same clusters, only a few of these

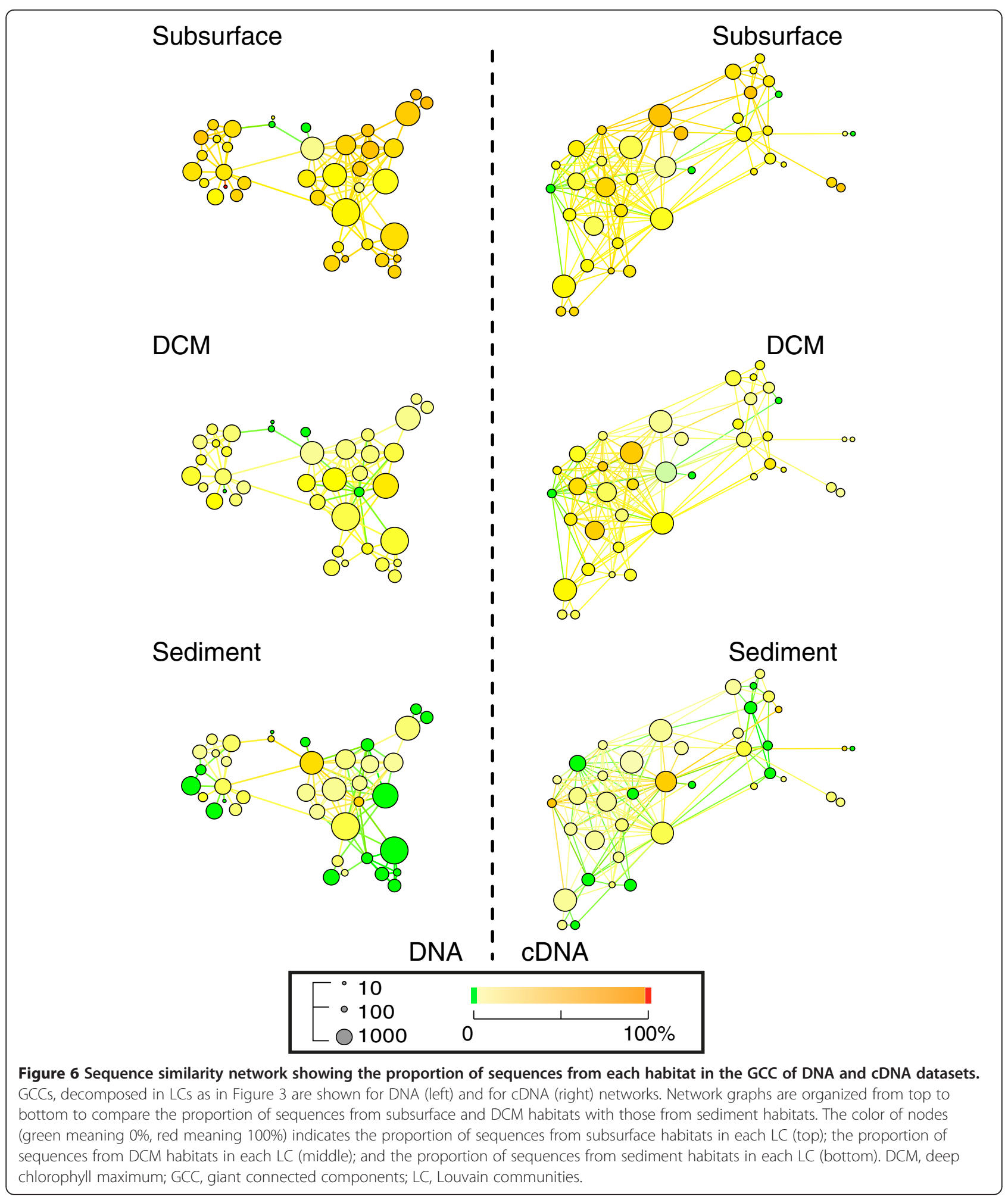


clusters also include sediment sequences. In Figure 6 this observation is highlighted by the large number of LCs which are non-green in both the top (subsurface) and middle (DCM) graphs, compared to the increasing number of green LCs in the bottom (sediment) graphs.

This result of contrasting sequences from sediments and water column also occurs for conspecific ciliates (defined here as groups of ciliates with $\geq 97 \%$ similar sequences). Using a sequence similarity threshold of $97 \%$ for CC analyses, we found that $50 \%$ of all sediment cDNA components are exclusive to this habitat, while only $4 \%$ and $9 \%$ are exclusive to the subsurface and the DCM (Additional file 9: Figure S5). A similar trend was observed in DNA components, of which 30\% were sediment-exclusive, $12 \%$ subsurface-exclusive and $7 \%$ DCM-exclusive (Additional file 9: Figure S5). Habitat specialization of marine protistan species at an even closer geographical proximity was also reported by Orsi and colleagues [50], who could relate their findings to a distinct geochemical gradient. In comparison, our results show a less pronounced level of habitat specialization along the water column, which we explain by the lack of a geochemical gradient and, thus, the less exigency for organisms to adapt. On the other hand, the high level of ciliate organisms which are specialized to sediment habitats is not unexpected, because a psammophilic life style requires different adaptations than a planktonic life style [73]. In a morphology-based approach combining publicly available ciliate data of 17 globally distributed marine benthic sampling sites, Azovsky and Mazei [74] could show that one quarter of the observed ciliate species were only detected at one single sample site and every second species occurred at not more than three different sites. Likewise, an environmental SSU-rDNA study [75] revealed that geographical structures at the sea-floor can act as biogeographical barriers and lead to distinctive benthic microbial communities. A more fragmented distribution of locally restricted benthic ciliate species, induced by geographical structures, such as the Strait of Gibraltar, could explain the comparably high level of exclusive CCs from the sediment observed in the current network approach.

\section{Conclusions}

\section{Network analysis in microbial biogeography}

We present network analyses as highly efficient tools to exploit massive HTS datasets and identify novel diversity. Further, network analyses appear as a useful means to address fundamental subjects in ecology using environmental diversity inventories. Such subjects include the analyses of microbial distribution patterns that allow us to draw conclusions about the underlying biological causes. For instance, while closely related ciliate sequences grouped together in our networks, the in-depth analyses of these groups showed that they are structured with regard to habitat or geographic location. With a broad ecological perspective, this can be interpreted as taxonomically closely related groups (depending on the resolution of sequence similarity we choose) which form distinct subcommunities in different habitats or at different geographic locations in European coastal waters. At the same time, we also find highly adapted groups which are restricted to one specific habitat or one location. This is similar to patterns observed in animals and plants [76] and confirms observations on ciliate dispersal patterns from other oceanographic areas [49,50]. Using network analyses of molecular markers, important theories can thus be tested: the technology for massive data production at an affordable price is in place, and powerful inclusive methods are available.

As we integrated data from multiple samples into the same comparative context, we must also conclude that surveying ciliate diversity appears more as a challenge than ever. The restriction of most taxa to one or a few different geographic locations and habitats, supported by all network estimates and for both DNA and cDNA sequences, means that we may have to bury our hopes to exhaust the ciliate (and most likely the microbial) diversity of an environment using available 'universal' primers and local in-depth sequencing [62]. Network analyses of sequence data can still contribute to explore such a structured vast microbial diversity. Sequence similarity networks provide all the tools needed to detect exclusively environmental clusters (CC or LC) and/or groups of sequences which are distant from previously described diversity. Alignment of groups of novel, peripheral, environmental sequences thus revealed by network analyses can guide the design of new sets of primers which will be useful to expand our knowledge of microbial diversity, moving it further away from the current status quo and its logical starting point: the sequences of cultured organisms.

The creation of distinct primers should compensate for the inherent tendency of recent environmental rDNA surveys whose findings gravitate around the same groups of organisms [62]. We encourage readers who want more than 'more of the same' from diversity surveys, and to enhance the scale at which ecological theories about microbial diversity can be tested, to experience this kind of inclusive network-based strategy.

\section{Methods}

\section{Constitution of the dataset}

A first reference database of ciliate sequences was built including 308 V4 SSU-rDNA sequences from GenBank. Only sequences from cultured and morphologically identified isolates were selected. Additionally a second reference database of 82,560 environmental ciliate 
SSU-rDNA sequences from earlier diversity studies was generated from GenBank. Moreover, environmental samples were collected at eight European marine coastal sites as part of the biodiversity of marine eukaryotes project (BioMarKs [48]). Information about sampling procedure, filter preparation, extraction of nucleic acids, sequencing strategy, sequence quality check and taxonomic assignment (on the phylum level) of the samples can be found in Logares et al. (2014) [18]. Stringent cleaning options were used (exact primer matching, removing low quality score sequences using sliding windows of $50 \mathrm{bp}$, and chimera checking) to guarantee the quality and reliability of the final dataset. Each sequence was labelled based on its origin (cultured organism, earlier environmental project, BioMarKs environmental sample), depth in the water column (subsurface, DCM, sediment), and sampling site of the BioMarKs project [48]. In all environmental datasets, only sequences which could be assigned to a culture sequence belonging to the phylum Ciliophora and had at least $300 \mathrm{bp}$ in length were considered, amounting to 85,482 BioMarKs ciliate V4 SSU-rDNA sequences. Furthermore, datasets were dereplicated (when identical, only unique sequences were kept for each sampling site in the BioMarKs database), which resulted in a final dataset of 25,842 unique BioMarKs sequences $(8,931$ DNA and 16,911 cDNA sequences, respectively), 308 ciliate culture sequences and 928 ciliate sequences of earlier environmental studies (listed in Additional file 1: Table S1). Sequence data used in this analysis are publicly available at Figshare $[59,77]$. Additionally, we deposited one table listing all BioMarKs ciliate sequences and their respective properties [78] and one table listing all cultured ciliate reference sequences [78]. Two fasta files that include all novel ciliate diversity sequences as listed in Figure 4 are also available and may be used to design novel primers (DNA fasta file: [60]; cDNA fasta file: [61]). For better understanding of the deposited data, we also provide a manual on how to navigate in our files [79].

\section{Graph construction}

Two types of sequence similarity networks (Figure 1) were constructed in this study to compare the consilience between the information within BioMarKs DNA sequences on the one hand and RNA sequences on the other hand. We used (1) a network with BioMarKs DNA plus reference sequences (10,167 sequences) and (2) a network with BioMarKs cDNA plus reference sequences $(18,147$ sequences). In both cases, sequences were used as an input file for EGN [80] choosing the following options: BLASTn E-value <1e-5; minimum hit identity threshold 60\%; minimum hit length $40 \%$ of the smallest homolog. This protocol excluded singletons and resulted in disconnected networks at various sequence similarity thresholds (85\%, 90\%, 95\%, 96\%, 97\%, 98\%, 99\%). Meaning that in a network with a sequence similarity threshold of $97 \%$, two nodes (where each node represents one sequence from the dereplicated DNA or cDNA dataset, respectively) are connected by an edge only if they share a sequence similarity of at least $97 \%$ (Figure 1A). As a consequence, sequence similarity networks provide a first clustering, producing connected components (CCs), that is, sets of connected sequences isolated from the rest of the graph (Figure 1A). A second level of sequence clustering was achieved using the Louvain method [46] (default parameters, first level, for example, the most fine-grained resolution) (Figure 1C). This method identifies densely connected nodes in a graph, and aggregates these nodes into clusters (Louvain communities (LCs)).

The EGN output which served as the basis for all subsequent network analyses in the context of our work is publicly available at Figshare [81].

\section{Graph display}

The largest connected component of the sequence similarity networks was found at $\geq 85 \%$ similarity and its LCs were displayed using Gephi [82] (Yifan $\mathrm{Hu}$ multilevel layout). Each LC is represented by a supernode. Two LCs are connected to another, when there is at least one connection between the two sets of sequences belonging to that pair of LC (Figures 3 and 6). The number of sequences in a LC is represented by the supernode size. LCs were colored based on their proportion of sequences with a label of interest (for example, cultured or environmental sequence, habitat affiliation) to display the structure of genetic diversity of ciliates (Figures 3 and 6).

\section{Graph analyses}

Clusters exclusively comprising sequences with the same label (for example, BioMarKs sequences or cultured ciliate sequences) were quantified. Using Igraph library scripts [83] in the $\mathrm{R}$ statistical computing environment [84], we applied two measures of dissimilarity between BioMarKs sequences, environmental sequences obtained in previous projects, and sequences from cultured ciliates. First, we used the minimal shortest path between all pairs of nodes of interest, expressed in number of edges (Table 1, Figure 2D); in other words, the minimal number of edges that must be crossed to connect any environmental node (that is, a BioMarKs sequence or a sequence from a previous environmental project) and its closest node of a cultured ciliate sequence (infinite when no such path existed). Second, we used the closeness of environmental nodes and nodes from cultured ciliates in our graphs (Table 1, Figure 2D). This closeness quantifies the centrality of sequences in the graph: more 
peripheral sequences are more different from most other sequences of the dataset. The distributions of closeness values for these groups of nodes were compared using the Kolmogorov-Smirnov test $(P<0.05$ and $P<0.01)$ with the unilateral option (test 1: cultured sequences versus sequences from former environmental studies; test 2 : cultured sequences versus all environmental sequences (former environmental studies plus BioMarKs)).

\section{Testing the global dispersal theory}

Two distinct measures were used to test whether all groups of ciliates with similar V4 DNA or cDNA sequences were found at all sampling sites. First, assortativity-a measure for the tendency of nodes with the same label (or of nodes without that label) to preferentially connect with one another in the graph-was computed for each label for each CC and LC. Assortativity was defined as in Newman (2003) [47], for two categories (nodes of the targeted label-for example, a given habitat-and other nodes). An assortativity coefficient $r=0$ means that edges between the two categories are distributed randomly between the two categories. A positive coefficient indicates that nodes of the same category (that is, same depth or location) tend to be linked together, while a negative one indicates that nodes of different categories tend to be linked together. Therefore, under total endemism, patterns as in Figure $2 \mathrm{~A}$ are expected in a graph. Sequences from a given depth or location cluster preferentially together and fall within the same exclusive region of the graph. By contrast, in the case of a cosmopolitan distribution of ciliates, similar sequences can be found in all depths and locations, producing a pattern such as that of Figure 2B.

Statistical significance of the assortativity values was assessed by randomly shuffling the labels over all sequences for each CC and LC while keeping the same network topology. Assortativity was computed for each label before and after the shuffling. Distributions of original and randomized assortativity values were compared with a Kolmogorov-Smirnov test $(P<0.05)$ to test whether observed assortativity values were significantly greater than by chance.

Second, for each cluster of sequences in our $\geq 99 \%$ similarity networks, likely grouping sequences of the same ciliate species, we plotted the abundance of that cluster (measured as the number of sequences), the occurrence of that cluster (measured as the number of sampling sites at which these sequences were detected), and the evenness of that cluster, measured by the Simpson index (SI) $\left[S I=1-\sum_{i=1}^{\text {objects }} p_{i}^{2}\right]$ in which $p_{\mathrm{i}}$ corresponds to the proportional abundance of units (here: sequences) counted in object $i$ (here: each sampling site) [85]. The value of SI ranges between 0 and an upper limit of $1-1 / \mathrm{N}$ ( $\mathrm{N}$ being the total number of objects). A cluster of sequences which is perfectly homogeneously distributed among the sampling sites will reach a SI close to 1 .

\section{Additional files}

Additional file 1: Table S1. Publicly available SSU-rDNA sequence data included into the environmental reference database. The table shows which studies had been included into the environmental reference database of our sequence similarity network approach. The first column gives the number of V4-SSU-rDNA sequences of each study which fulfilled our requirements and were thus incorporated into the environmental reference database.

Additional file 2: Figure S1. Proportion of CCs (above) and LCs (below) in DNA and cDNA networks which exclusively contained BioMarks sequences. DNA data are shown in red, cDNA data in blue. Composition of each CC and LC was analyzed at all sequence similarity thresholds to detect references from cultured ciliate sequences and sequences of former environmental studies. Proportion of CCs and LCs displayed here only contain sequences of BioMarks project. The more inclusive the threshold (that is, the lower the sequence similarity), the fewer CCs and LCs can be detected. Thus, the proportion of CCs and LCs exclusively containing BioMarks sequences decreases with lower similarity thresholds. In comparison to DNA networks, a higher proportion of exclusive CCs and LCs can be found in the cDNA networks, except for the most inclusive similarity threshold ( $\geq 85 \%$ ).

Additional file 3: Figure S2. Network path analyses of CCs. For each environmental node at all sequence similarity thresholds, the shortest path to a reference node (node of a cultured ciliate) has been calculated. Shortest path analyses describe the minimum number of edges linking the initial node with the target node. Distance of ' 1 ' to a reference node means that the environmental node is directly connected to the reference node (cultured ciliate). Distance of 'inf' (means infinite) to a reference node means that the environmental node is in a CC without any reference node (cultured ciliate), hence no shortest path can be calculated. The proportion of environmental nodes for which a path length of 'inf' is reported, decreases with the sequence similarity threshold. Numbers on top of each plot indicate the abundance of CCs regarding the sequence similarity threshold.

Additional file 4: Figure S3. Network path analyses of LCs. For each environmental node at all sequence similarity thresholds, the shortest path to a reference node (node of a cultured ciliate sequence) has been calculated. Shortest path analyses of LCs revealed similar patterns as the shortest path analyses of CCs (Additional file 3: Figure S2).

Additional file 5: Table S2. Kolmogorov-Smirnov (KS) tests comparing closeness distributions in DNA networks. At all sequence similarity thresholds and for both CCs and LCs two independent KS-tests were performed: 1) to test if the closeness of nodes of cultured ciliates $\left(C_{\text {cultured }}\right)$ was higher than the closeness of nodes from former environmental studies before 454 sequencing ( $C_{\text {former Env }}$ ) and 2 ) to test if the closeness of $C_{\text {cultured }}$ was higher than the closeness of all environmental nodes ( $C_{\text {Env }}$ : nodes from former environmental studies plus BioMarks nodes). Closeness can be understood as a measurement of centrality in a network (that is, the higher the closeness, the more central the nodes will be located). For all but one case it could be confirmed that $C_{\text {cultured }}$ was significantly higher than $C_{\text {former Env }}(P<0.05$ or $P<0.01)$. Compared to $C_{\text {Env, }} C_{\text {cultured }}$ was always significantly higher.

Additional file 6: Table S3. KS-tests comparing closeness distributions in CDNA networks. Results and patterns of the KS-tests on closeness in DNA networks (Additional file 5: Table S2) are confirmed.

Additional file 7: Table S4. KS-test comparing the distribution of assortativity in gene similarity networks. Assortativity describes the tendency of nodes to be connected to nodes of the same label. Two independent groups of labels (three habitats, eight locations) were tested for DNA and CDNA networks at each sequence similarity threshold. Using a one-sided KS-test we analyzed if the distribution of assortativity values 
of each respective label was significantly greater $(P<0.05)$ than expected by chance. Thus the nodes of the respective label were more likely to connect with each other than to connect to nodes of another label.

Additional file 8: Figure S4. Three-dimensional plots displaying abundance, occurrence and evenness of sequences in LCs. Plots are based on DNA (above) and cDNA (below) networks at the most exclusive sequence similarity threshold ( $\geq 99 \%$ ). Each dot (370 for DNA; 670 for CDNA) represents one LC.

Additional file 9: Figure S5. Composition of CC in DNA (above) and cDNA (below) gene similarity networks. Each CC at each similarity threshold was analyzed to highlight the proportion of nodes, which derived from the three investigated habitats in this study (Subsurface, DCM, Sediment). The graphs show the proportion of all CCs, which exclusively consist of nodes of the same habitat. In all networks the highest proportion of exclusive CCs was found for sediment habitats (orange), in some networks providing more than $50 \%$ of all CCs. The proportion of DCM (green) and subsurface (blue) CCs never reached more than $15.4 \%$ and $16.4 \%$, respectively, of the total number of CCs in the respective network. In the DNA networks an increase of CCs harboring exclusively nodes of DCM habitats could be observed with decreasing sequence similarity. The reason for this is a few exclusive DCM CCs which do not merge with any other CC although the threshold gets more inclusive and fewer CCs in total can be found. Consequently - as the reported values are proportions, not absolute numbers-more CCs can be detected in proportion to the total number of CCs.

\section{Competing interests}

The authors declare that they have no competing interests.

\section{Authors' contributions}

TS and EB conceived and guided the study; DF and LB performed network analyses; DF, LB, TS and EB interpreted and put data into scientific context; $\mathrm{MD}$ constructed and provided the V4-database; DF, LB, SK, MD, TS and EB wrote the manuscript; $L B, M D$, SR and SA performed sampling; LB, SK, SA, PL and EB performed bioinformatical analyses. All authors read and approved the final manuscript.

\section{Authors' information}

Dominik Forster and Lucie Bittner: share first authorship.

Thorsten Stoeck and Eric Bapteste: share senior authorship.

\section{Acknowledgements}

We thank PA Jachiet for technical help. We are grateful to the CNRS-UPMC ABiMS bioinformatics platform (http://abims.sb-roscoff.fr) for providing computational resources and support. The BioMarks 454 sequence data were generated as part of a study supported by the EU-FP7 ERA-net program BiodivERsA, under the project BioMarks (2008-6530). We thank all members of the BioMarks consortium who participated in field sampling and data generation. We also appreciate the support of the Research Unit BioComp at the University of Kaiserslautern, funded by the Research Initiative of Rhineland-Palatinate. This work was supported by a grant from Stipendienstiftung Rheinland-Pfalz to D.F.; a grant from University of Kaiserslautern to L.B.; Deutsche Forschungsgemeinschaft (DFG, grant \# DU1319/1-1) to M.D.; DFG (grants \# STO414/3-1, STO414/3-2 and STO414/11-1) to T.S., and by a grant from Region Ile-de-France (DIM Malinf) to S.K. E.B. is funded by the European Research Council under the European Community's Seventh Framework Programme FP7 (2007-2013 Grant Agreement n615274). We greatly appreciate the comments of three anonymous reviewers for their constructive comments on our manuscript.

\section{Author details}

'Department of Ecology, University of Kaiserslautern, Erwin-Schrödinger-Straße 14, Kaiserslautern D-67633, Germany. ${ }^{2}$ CNRS, FR3631, Institut de Biologie Paris-Seine, Paris F-75005, France. ${ }^{3}$ Sorbonne Universités, UPMC Univ Paris 06, Institut de Biologie Paris-Seine (IBPS), Paris F-75005, France. ${ }^{4} \mathrm{CNRS}$, UMR7138, Institut de Biologie Paris-Seine, Paris F-75005, France. ${ }^{5}$ CNRS, UMR 7144, Station Biologique de Roscoff, Place Georges Teissier, Roscoff F-29680, France. ${ }^{6}$ Sorbonne Universités, UPMC Univ Paris 06, UMR 7144, Station Biologique de Roscoff, Place Georges Teissier, Roscoff F-29680, France.
Received: 12 August 2014 Accepted: 28 January 2015

Published online: 24 February 2015

\section{References}

1. Falkowski PG, Fenchel T, Delong EF. The microbial engines that drive Earth's biogeochemical cycles. Science. 2008;320:1034-9.

2. Keller M, Zengler K. Tapping into microbial diversity. Nat Rev Microbiol. 2004;2:141-50

3. Staley JT, Reysenbach AL. Biodiversity of microbial life: foundation of earth's biosphere. New York: Wiley-Liss, Inc; 2001.

4. Finlay BJ, Esteban GF, Olmo JL, Tyler PA. Global distribution of free-living microbial species. Ecography. 1999;22:138-44.

5. Finlay BJ. Global dispersal of free-living microbial eukaryote species. Science. 2002;296:1061-3.

6. Foissner W. Biogeography and dispersal of micro-organisms: a review emphasizing protists. Acta Protozool. 2006;45:111-36.

7. Foissner W, Chao A, Katz LA. Diversity and geographic distribution of ciliates (Protista: Ciliophora). Biodivers Conserv. 2008;17:345-63.

8. Bass D, Richards TA, Matthai L, Marsh V, Cavalier-Smith T. DNA evidence for global dispersal and probable endemicity of protozoa. BMC Evol Biol. 2007;7:162.

9. Green JL, Holmes AJ, Westoby M, Oliver I, Briscoe D, Dangerfield M, et al. Spatial scaling of microbial eukaryote diversity. Nature. 2004;432:747-50.

10. Green J, Bohannan BJ. Spatial scaling of microbial biodiversity. Trends Ecol Evol. 2006;21:501-7.

11. Martiny JB, Bohannan BJ, Brown JH, Colwell RK, Fuhrmann JA, Green JL, et al. Microbial biogeography: putting microorganisms on the map. Nat Rev Microbiol. 2006;:102-12.

12. Dawson SC, Pace NR. Novel kingdom-level eukaryotic diversity in anoxic environments. Proc Natl Acad Sci U S A. 2002;99:8324-9.

13. Stoeck T, Hayward B, Taylor GT, Varela R, Epstein SS. A multiple PCR-primer approach to access the microeukaryotic diversity in environmental samples. Protist. 2006;157:31-43.

14. Medinger R, Nolte V, Pandey RV, Jost S, Ottenwälder B, Schlötterer C, et al. Diversity in a hidden world: potential and limitation of next-generation sequencing for surveys of molecular diversity of eukaryotic microorganisms. Mol Ecol. 2010;19:32-40.

15. Schuster SC. Next-generation sequencing transforms today's biology. Nat Methods. 2008:5:16-8.

16. Bittner L, Gobet A, Audic S, Romac S, Egge ES, Santini S, et al. Diversity patterns of uncultured Haptophytes unravelled by pyrosequencing in Naples Bay. Mol Ecol. 2013;22:87-101.

17. Dunthorn M, Otto J, Berger SA, Stamatakis A, Mahé F, Romac S, et al. Placing environmental next-generation sequencing amplicons from microbial eukaryotes into a phylogenetic context. Mol Biol Evol. 2014:31:993-1009.

18. Logares R, Audic S, Bass D, Bittner L, Boutte C, Christen R, et al. Patterns of rare and abundant marine microbial eukaryotes. Curr Biol. 2014;24:813-21.

19. Sogin ML, Morrison HG, Huber JA, Welch DM, Huse SM, Neal PR, et al. Microbial diversity in the deep sea and the underexplored "rare biosphere". Proc Natl Acad Sci U S A. 2006;103:12115-20.

20. Stoeck T, Behnke A, Christen R, Amaral-Zettler L, Rodriguez-Mora MJ, Chistoserdov A, et al. Massively parallel tag sequencing reveals the complexity of anaerobic marine protistan communities. BMC Biol. 2009;7:72.

21. Stoeck T, Bass D, Nebel M, Christen R, Jones MD, Breiner HW, et al. Multiple marker parallel tag environmental DNA sequencing reveals a highly complex eukaryotic community in marine anoxic water. Mol Ecol. 2010;19:21-31.

22. Pawlowski J, Lecroq B. Short rDNA barcodes for species identification in Foraminifera. J Eukaryot Microbiol. 2010;57:197-205.

23. Behnke A, Engel M, Christen R, Nebel M, Klein RR, Stoeck T. Depicting more accurate pictures of protistan community complexity using pyrosequencing of hypervariable SSU rRNA gene regions. Env Microb. 2010;13:340-9.

24. Dunthorn M, Klier J, Bunge J, Stoeck T. Comparing the hyper-variable V4 and $\mathrm{V} 9$ regions for assesment of ciliate environmental diversity. J Eukaryot Microbiol. 2012;59:185-7.

25. Mahé F, Mayor J, Bunge J, Chi J, Siemensmayer T, Stoeck T, et al. Comparing high-throughput platforms for sequencing the V4 region of SSU-rDNA in environmental microbial eukaryotic diversity surveys. J Euk Microbiol. 2014; doi:10.1111/jeu.12187. 
26. Stern RF, Andersen RA, Jameson I, Küpper FC, Coffroth MA, Vaulot D, et al. Evaluating the ribosomal internal transcribed spacer (ITS) as a candidate dinoflagellate Barcode marker. PLoS One. 2012;7:e42780.

27. Schoch CL, Seifert KA, Huhndorf S, Robert V, Spouge JL, Levesque CA, et al. Nuclear ribosomal internal transcribed spacer (ITS) region as a universal DNA barcode marker for Fungi. Proc Natl Acad Sci U S A. 2012;109:6241-6.

28. Huse SM, Dethlefsen L, Huber JA, Welch DM, Relman DA, Sogin ML. Exploring microbial diversity and taxonomy using SSU rRNA hypervariable tag sequencing. PLoS Genet. 2008;4:e1000255.

29. Bates ST, Berg-Lyons D, Caporaso JG, Walters WA, Knight R, Fierer N. Examining the global distribution of dominant archaeal populations in soil. ISME J. 2010;5:908-17.

30. Alvarez-Ponce D, Lopez P, Bapteste E, Mclnerney JO. Gene similarity networks provide tools for understanding eukaryote origins and evolution. Proc Natl Acad Sci U S A. 2013;110:1594-603.

31. Bapteste E, Lopez P, Bouchard F, Baquero F, Mclnerney JO, Burian RM. Evolutionary analyses of non-genealogical bonds produced by introgressive descent. Proc Natl Acad Sci U S A. 2012;109:18266-72.

32. Bapteste E, Bicep C, Lopez P. Evolution of genetic diversity using networks: the human gut microbiome as a case study. Clin Microbiol Infect. 2012:18:40-3.

33. Bapteste $E$, van lersel L, Janke A, Kelchner S, Kelk S, Mclnerney JO, et al. Networks: expanding evolutionary thinking. Trends Genet. 2013;29:439-41.

34. Bittner L, Halary S, Payri C, Cruaud C, de Reviers B, Lopez P, et al. Some considerations for analyzing biodiversity using integrative metagenomics and gene networks. Biol Direct. 2010;5:47.

35. Lynch MD, Bartram AK, Neufeld JD. Targeted recovery of novel phylogenetic diversity from next-generation sequence data. ISME J. 2012;6:2067-77.

36. Faust K, Raes J. Microbial interactions: from networks to models. Nat Rev Microbiol. 2012;10:538-50.

37. Chaffron S, Rehrauer H, Pernthaler J, von Mering C. A global network of coexisting microbes from environmental and whole-genome sequence data. Genome Res. 2010;20:947-59.

38. Atkinson HJ, Morris JH, Ferrin TE, Babbitt PC. Using sequence similarity networks for visualization of relationships across diverse protein superfamilies. PLoS One. 2009;4:e4345

39. Dagan T, Artzy-Randrup Y, Martin W. Modular networks and cumulative impact of lateral transfer in prokaryote genome evolution. Proc Natl Acad Sci U S A. 2008;105:10039-44.

40. Halary S, Leigh JW, Cheaib B, Lopez P, Bapteste E. Network analyses structure genetic diversity in independent genetic worlds. Proc Natl Acad Sci U S A. 2010;107:127-32.

41. Jachiet PA, Pogorelcnik R, Berry A, Lopez P, Bapteste E. MosaicFinder: identification of fused gene families in sequence similarity networks. Bioinformatics. 2013;29:837-44.

42. Medini D, Covacci A, Donati C. Protein homology network families revea step-wise diversification of Type III and Type IV secretion systems. PLoS Comp Biol. 2006;2:e173.

43. Song N, Joseph JM, Davis GB, Durand D. Sequence similarity network reveals common ancestry of multidomain proteins. PLoS Comp Biol. 2008;4:e1000063.

44. Berger SA, Krompass D, Stamatakis A. Performance, accuracy and web server evolutionary placement of short sequence reads under maximum likelihood. Syst Biol. 2011;60:291-302.

45. Matsen FA, Kodner RB, Armbrust EV. pplacer: linear time maximum-likelihood and Bayesian phylogenetic placement of sequences onto a fixed reference tree. BMC Bioinformatics. 2010;11:538

46. Blondel VD, Guillaume JL, Lambiotte R, Lefebvre E. Fast unfolding of communities in large networks. J Stat Mech. 2008;10:P10008.

47. Newman ME. Mixing patterns in networks. Phys Rev E Stat Nonlin Soft Matter Phys. 2003;67:026126.

48. Biodiversity of Marine Eukaryotes. http://biomarks.eu.

49. Edgcomb V, Orsi W, Bunge J, Jeon S, Christen R, Leslin C, et al. Protistan microbial observatory in the Cariaco Basin: Caribbean I Pyrosequencing vs Sanger insights into species richness. ISME J. 2011;5:1344-56.

50. Orsi W, Edgcomb V, Jeon S, Leslin C, Bunge J, Taylor GT, et al. Protistan microbial observatory in the Cariaco Basin: Caribbean II Habitat specialization. ISME J. 2011;5:1357-73.
51. Orsi W, Song YC, Hallam S, Edgcomb V. Effect of oxygen minimum zone formation on communities of marine protists. ISME J. 2012;6:1586-601.

52. Dolan JR, Landry MR, Ritchie ME. The species-rich assemblages of tintinnids (marine planktonic protists) are structured by mouth size. ISME J. 2013;7:1237-43.

53. Dolan JR, Pierce RW. Diversity and distributions of Tintinnids. In: Dolan JR, Montagnes DJ, Agatha S, Coats DW, Stoecker DK, editors. The biology and ecology of Tintinnid ciliates: models for marine plankton. 1st ed. Hoboken: Wiley-Blackwell; 2013. p. 214-43.

54. Junker B, Schreiber F. Analysis of biological networks. Hoboken: John Wiley \& Sons; 2008.

55. Erdõs $P$, Rényi $A$. On the evolution of random graphs. Publ Math Inst Hung Acad Sci. 1960;5:17-61

56. Newman ME. A measure of betweenness centrality based on random walks. Soc Netw. 2005;27:39-54

57. Pedrós-Alió C. Marine microbial diversity: can it be determined. Trends Microbiol. 2006;14:257-63.

58. Dunthorn M, Stoeck T, Clamp J, Warren A, Mahé F. Ciliates and the rare biosphere: a review. J Euk Microbiol. 2014;61:404-9.

59. Figshare. http://figshare.com.

60. Novel ciliate diversity sequences (DNA). http://dx.doi.org/10.6084/m9. figshare.1266267.

61. Novel ciliate diversity sequences (cDNA). http://dx.doi.org/10.6084/m9. figshare.1266266.

62. Jeon S, Bunge J, Leslin C, Stoeck T, Hong S, Epstein S. Environmental rRNA inventories miss over half of protistan diversity. BMC Microbiol. 2008;8:222.

63. Makino K, Uno T. New algorithms for enumerating all maximal cliques. In: Hagerup T, Katajainen J, editors. Algorithm theory-SWAT 2004: 8-10 July 2004; Humlebæk. Berlin, Heidelberg: Springer Verlag; 2004. p. 260-72.

64. Agatha S. Global diversity of aloricate Oligotrichea (Protista, Ciliophora, Spirotricha) in marine and brackish sea water. PLoS One. 2011;6:e22466.

65. Dunthorn M, Stoeck T, Wolf K, Breiner HW, Foissner W. Diversity and endemism of ciliates inhabiting neotropical phytotelmata. Syst Biodivers. 2012;10:195-205.

66. Forster D, Behnke A, Stoeck T. Meta-analyses of environmental sequence data identify anoxia and salinity as parameters shaping ciliate communities. Syst Biodivers. 2012;10:277-88.

67. Lozupone CA, Knight R. Global patterns in bacterial diversity. Proc Natl Acad Sci U S A. 2007;104:11436-40.

68. Revsbech NP, Sørensen J, Blackburn H, Lomholt JP. Distribution of oxygen in marine sediments, measured with microelectrodes. Limnol Oceanog. 1980:25:403-11.

69. Jenkins DG, Brescacin CR, Duxbury CV, Elliott JA, Evans JA, Grablow KR, et al. Does size matter for dispersal distance? Global Ecol Biogeogr. 2007; 16:415-25.

70. Papke RT, Ward DM. The importance of physical isolation to microbial diversification. FEMS Microbiol Ecol. 2004;48:293-303.

71. Fierer N. Microbial biogeography: patterns in microbial diversity across space and time. In: Zengler K, editor. Accessing uncultivated microorganisms: from the environment to organisms and genomes and back. Washington DC: ASM Press; 2008. p. 95-115.

72. Gibbons SM, Caporaso JG, Pirrung M, Field D, Knight R, Gilbert JA. Evidence for a persistent microbial seed bank throughout the global ocean. Proc Natl Acad Sci U S A. 2013;110:4651-5.

73. Jørgensen BB, Boetius A. Feast and famine - microbial life in the deep-sea bed. Nat Rev Microbiol. 2007;5:770-81.

74. Azovsky A, Mazei Y. Do microbes have macroecology? Large-scale patterns in the diversity and distribution of marine benthic ciliates. Global Ecol Biogeogr. 2013;22:163-72.

75. Scheckenbach F, Hausmann K, Wylezich C, Weitere M, Arndt H. Large-scale patterns in biodiversity of microbial eukaryotes from the abyssal sea floor. Proc Natl Acad Sci U S A. 2010;107:115-20.

76. Lomolino MV, Riddle BR, Brown JH. Biogeography. 3rd ed. Sunderland: Sinauer Associates, Inc; 2006.

77. V4 ciliate sequence dataset. http://dx.doi.org/10.6084/m9.figshare.1263932.

78. Supporting information to V4 ciliate sequences dataset. http://dx.doi.org/ 10.6084/m9.figshare.1264009.

79. Manual for deposited files. http://dx.doi.org/10.6084/m9.figshare.1264013.

80. Halary S, Mclnerney JO, Lopez P, Bapteste E. EGN: a wizard for construction of gene and genome similarity networks. BMC Evol Biol. 2013;13:146

81. Network analyses input file. http://dx.doi.org/10.6084/m9.figshare.1263925. 
82. Bastian M, Heymann S, Jacomy M. Gephi: an open source software for exploring and manipulating networks. ICWSM. 2009;8:361-2. http://gephi.github.io/.

83. Csárdi G, Nepusz T. The igraph software package for complex network research. InterJournal Complex Systems. 2006;1695:1-9.

84. The R project for statistical computing. http://R-project.org.

85. Simpson EH. Measurement of diversity. Nature. 1949;163:688.

Submit your next manuscript to BioMed Central and take full advantage of:

- Convenient online submission

- Thorough peer review

- No space constraints or color figure charges

- Immediate publication on acceptance

- Inclusion in PubMed, CAS, Scopus and Google Scholar

- Research which is freely available for redistribution 